\title{
Design Considerations on a Dispersion Compensating Coaxial Fiber
}

\author{
F. D. Nunes \\ Departamento de Eletrônica e Sistemas, Centro de Tecnologia, \\ Universidade Federal de Pernambuco, \\ Caixa Postal 7800, 50711-970 Recife, PE, Brazil \\ H. F. da Silva and S. C. Zilio \\ Instituto de Física de São Carlos, Universidade de São Paulo, \\ Caixa Postal 369, 13560-970 São Carlos, SP, Brazil \\ Received September 30, 1997
}

\begin{abstract}
This work reports the performance of a dispersion compensating coaxial fiber as a function of its geometric parameters. Our analysis is carried out by solving the wave equation under the linearly polarized approximation, which leads to transcendental equations that provide the effective index of refraction. The highest efficiency at a fixed wavelength is achieved for a suitably chosen geometry and this choice is an important factor to determine the general shape of the wavelength-dependent dispersion curve.
\end{abstract}

\section{Introduction}

The development of Erbium-doped fiber amplifiers with gain at $1.55 \mu \mathrm{m}$ suggests the need of upgrading the existing $1.31 \mu \mathrm{m}$ optical fiber links for operation in that wavelength. This will bring the advantage of using unrepeted long-haul systems. However, transmission of high data rates over long distances using the already installed single mode fibers (SMF) requires the use of techniques to compensate the pulse spreading caused by positive chromatic dispersion. In order to accomplish this goal, many researchers have directed their attention to the use of dispersion compensating fibers (DCF) [1-4]. Peschel et al. [3] have recently shown that the supermodes of two dissimilar coupled planar waveguides may exhibit dispersion compensation and discussed the trade-off between group velocity dispersion (GVD) and bandwidth. Their theoretical analysis was carried out with the coupled wave guides model, where the modes in each isolated sub-structure are coupled through a coupling constant $\kappa$. Based on the idea of dissimilar waveguides, Thyagarajan et al. [4] extended this concept to a cylindrical geometry by performing a theo- retical analysis of a highly asymmetric four-layer fiber, whose refractive index profile is schematically shown in Fig. 1. It has four distinct regions: $\operatorname{rod}(0<r<a)$, gap $(a<r<b)$, barrier $(b<r<c)$ and clad $(r>c)$, and can be thought as composed of two sub-structures, namely rod [5] and tube [6], as indicated in the figure. Their analysis, carried out with a numerical technique proposed by Sharma et al. [7], indicates the possibility of large negative dispersion coefficients, $D$, with values as high as $5100 \mathrm{ps} /(\mathrm{nm} . \mathrm{km})$. The coupled mode theory used by Peschel et al. [3] was first studied by Boucouvalas [8] to describe four-layer coaxial fibers. The supermodes $\mathrm{HE}_{11}$ and $\mathrm{HE}_{12}$ of this kind of fibers are well described as combinations of $\mathrm{HE}_{11}$ modes of the two isolated sub-structures. More recently, Nunes et al. [9] presented a detailed theoretical study of different coaxial fibers, where the structure presented in Fig.1 is a particular case of them. In that work, the wave equation of the fibers were solved under the linearly polarized (LP) approximation [10], leading to transcendental equations from which the propagation constant, $\beta$, can be calculated. In this way, the effective index of refraction, $n(n=\beta / k$, where $k=2 \pi / \lambda$ and $\lambda$ is the 
wavelength), is obtained as well as universal dispersion curves for any fiber mode.

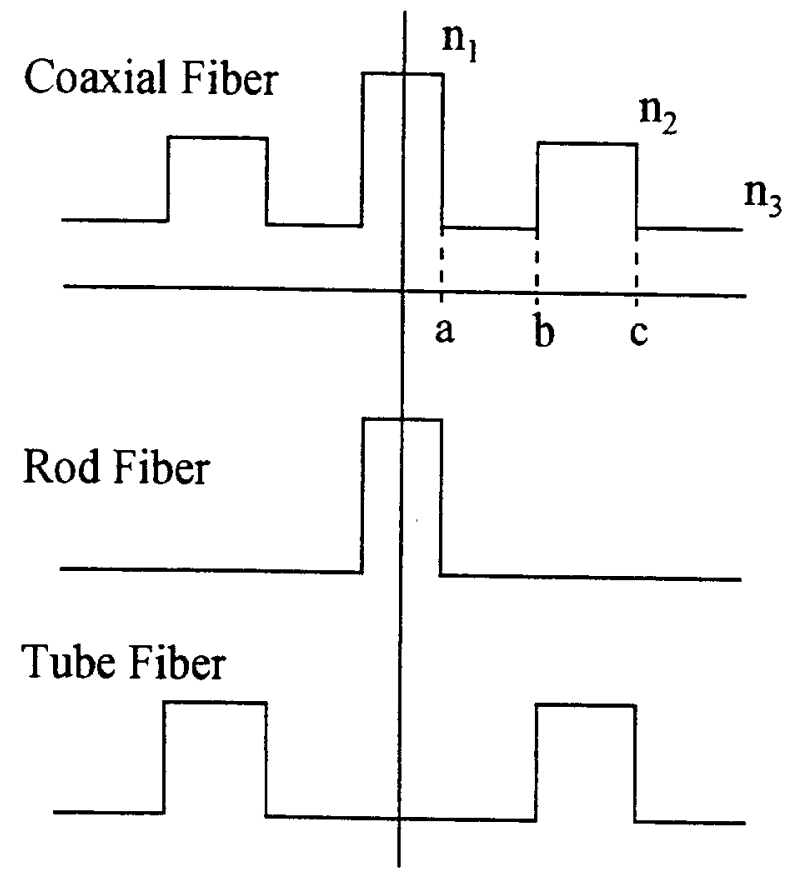

Figure 1. Refractive index radial profile of the dispersion compensating coaxial fiber. The sub-structures that compose the coaxial fiber (rod and tube) are also shown.

The present work employs the theoretical framework developed in ref. [9] to determine the dispersion coefficient of coaxial fibers, by performing a numerical calculation of the second order derivative of $n$. Therefore, the procedure adopted here is different from that using coupled modes [3], and also from the numerical approach developed in ref. [7]. The emphasis in this work is placed on the dependence of the dispersion coefficient on the geometrical parameters, namely, the rod radius, $a$, the outer gap radius, $b$, and the outer barrier radius, $c$. When they change, the depth and bandwidth of the wavelength-dependent dispersion coefficient can change significantly. There is a trade-off between the group velocity dispersion and bandwidth [3], and then it becomes important to determine the shape of the wavelength-dependent dispersion coefficient for different design situations.

\section{Fiber design and theoretical framework}

As mentioned in the previous section, the dispersion coefficient is calculated through the second derivative of the effective refractive index curve. These curves are obtained with the LP approximation for the coaxial fiber that has the profile shown in Fig. 1, and the resulting transcendental equations are those presented for the W1 structure (with $n_{2}=n_{4}$ ) in ref. [9]. The values of the refractive indices $n_{1}$ and $n_{2}$ are calculated by adding to the lower refractive index value, $n_{3}$, the same steps employed in ref. [4], namely , $\Delta_{1}=0.02$ and $\Delta_{2}=0.003$, where $\Delta_{i}=\left(n_{i}^{2}-n_{3}^{2}\right) /\left(2 n_{3}^{2}\right)$. The wavelength dependence is assumed to be the same for all refractive indices. $n_{3}$ was calculated with the wellknown Sellmeier equation: [10]

$$
n_{3}^{2}=1+\sum_{j=1}^{3} \frac{A_{j} \lambda_{j}^{2}}{\left(\lambda^{2}-\lambda_{j}^{2}\right)}
$$

with $A_{1}=0.69681388, A_{2}=0.40865177, A_{3}=$ $0.89374039, \lambda_{1}=0.070555513, \lambda_{2}=0.11765660$ and $\lambda_{3}=9.8754801$ [10]. Assuming the same parameters for all refractive indices does not affects the general trend of the results presented here, but a better approach is being searched for future work. Unfortunately, up to the author's knowledge, there are no available data to compare with the refractive index steps used here.

The dispersion coefficient is calculated according to [11]:

$$
D=-\frac{\lambda}{c} \frac{d^{2} n}{d \lambda^{2}}
$$

where $c$ is the speed of light in vacuum. Once $n$ is obtained from the transcendental equations, $D(\lambda)$ can be found by numerically determining its second derivative. The results were obtained with a Pentium $133 \mathrm{MHz}, 32$ Mb RAM personal computer, with the need of less than five minutes to generate each data file for $D$. The mode profiles can also be found with the theoretical approach given here, by substituting the propagation constant $\beta$ into the wave equation of the fibers.

\section{Numerical results and discussions}

Our results show that the dispersion enhancement occurs at the wavelength where the indices of the supermodes $\mathrm{HE}_{11}$ and $\mathrm{HE}_{12}$ approach each other, as shown in Fig. 2 for a coaxial fiber with $a=1 \mu \mathrm{m}, b=15 \mu \mathrm{m}$ and $c=22 \mu \mathrm{m}$. This is associated to the strong coupling between the two individual modes of the isolated sub-structures, rod and tube, which at that wavelength are phase matched $[3,4]$. According to eq. (2), the supermode $\mathrm{HE}_{11}$ will result in a negative dispersion, as required for compensation, while the mode $\mathrm{HE}_{12}$ 
will present a positive dispersion. When any geometric parameter changes, the wavelength where the phase matching occurs also changes, in addition to the shape of the dispersion curve. We first discuss the influence of the rod radius and of the outer gap radius in the dispersion curve, for a given value of the outer barrier radius. With the theoretical approach described previously [9], we found to be possible to obtain pairs of geometric parameters $(a, b)$ such that the dispersion occurs at the same wavelength, but the shape of the dispersion curve will depend on these parameters. This affects the desired compensation and constitutes the interest of the present work.

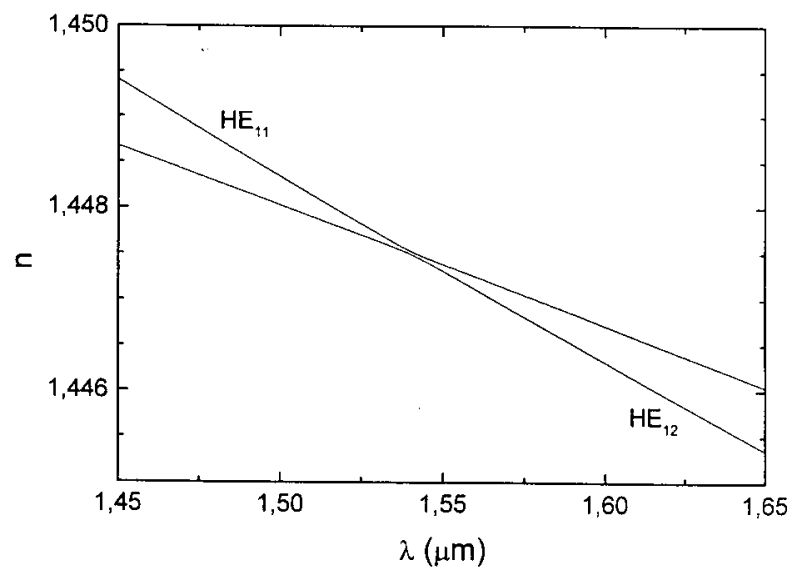

Figure 2. Indices of the supermodes $\mathrm{HE}_{11}$ and $\mathrm{HE}_{12}$ as a function of the wavelength.

Fig. 3 shows the dependence of $b$ on a such that the pairs $(a, b)$ will result in "maximum dispersion" at the wavelengths of interest for communication systems, $1.31 \mu \mathrm{m}$ and $1.55 \mu \mathrm{m}$. The value of $c$ is kept constant at $22 \mu \mathrm{m}$. The results indicate that any increase in the value of the rod radius can be compensated with a smaller value of the external gap region, as a way to produce the group velocity dispersion at the same wavelength. However, the behavior of the dispersion curve is much more sensitive to $a$ than to $b$, meaning that a small variation in a requires a much larger change in $b$, as can be seen in Fig. 3. We have also calculated wavelength-dependent dispersion curves for those pairs $(a, b)$ shown as solid circles in Fig. 3 and the results are presented in Fig. 4. The values of $D$ found with the present theoretical approach are in good agreement with those of Thyagarajan et al. [4] for $1.55 \mu \mathrm{m}$ when the same values of the fiber parameters are used $\left(a=1.0 \mu \mathrm{m}, b=15.2 \mu \mathrm{m}, c=22 \mu \mathrm{m}, \Delta_{1}=0.02\right.$ and $\left.\Delta_{2}=0.003\right)$. The maximum dispersion occurs at $a=1.01 \mu \mathrm{m}$ and $b=14.7 \mu \mathrm{m}$ for $1.55 \mu \mathrm{m}$, and $a=0.87$ $\mu \mathrm{m}$ and $b=15 \mu \mathrm{m}$ for $1.31 \mu \mathrm{m}$. Their values were found to be $D_{\max }^{1.55}=-4100 \mathrm{ps} /(\mathrm{nm} . \mathrm{km})$ and $D_{\max }^{1.31}=-21500$ $\mathrm{ps} /(\mathrm{nm} . \mathrm{km})$. Any increase or decrease of a results in a less efficient dispersion. Taking into account that the spectral half-width of a $10 \mathrm{ps}$ pulse is about $3.5 \times 10^{-4}$ $\mu \mathrm{m}$, Fig. 4 indicates that the bandwidth of the coaxial DCF is sufficiently large for the two wavelengths considered.

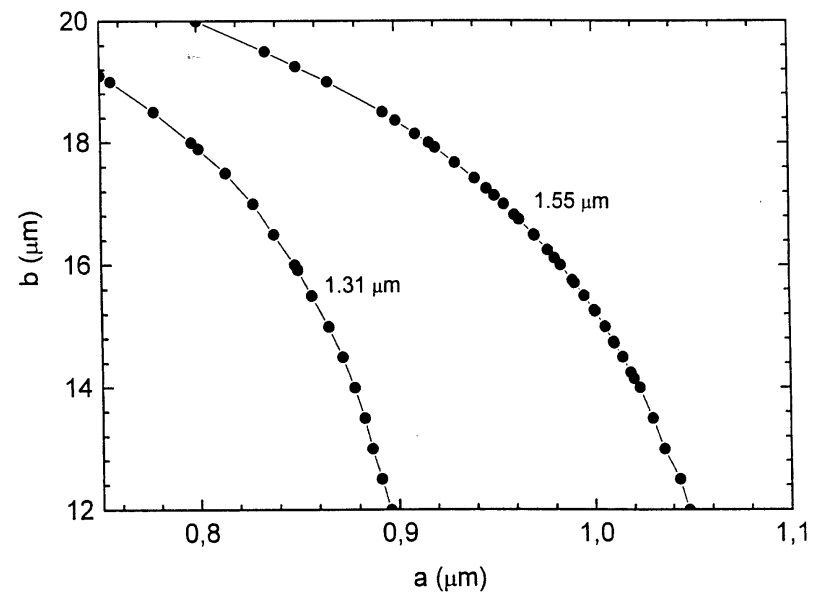

Figure 3. Pairs of geometric parameters $a$ and $b$ that provides the maximum dispersion at wavelengths of interest for communication systems, $1.31 \mu \mathrm{m}$ and $1.55 \mu \mathrm{m}$. The curves are just for a visual aid.

Next, we also consider the variation of the radius $c$, but in this case we restrict the discussion to $\lambda=1.55$ $\mu \mathrm{m}$. For each value of $c$ we evaluate those pairs $(a, b)$ that will produce a dispersion curve whose maximum efficiency is located at $1.55 \mu \mathrm{m}$. This procedure results in the family of curves shown in Fig. 5. The height of the dispersion dip at $1.55 \mu \mathrm{m}$ changes as we move along each of these curves, but we found that the highest dispersion always occurs for $a=1.02 \mu \mathrm{m}$ if $c$ is greater than $22 \mu \mathrm{m}$. This is schematically shown as the nearly vertical line in the figure. On the other hand, if we move upwards along this line, the value of the dispersion maximum increases steadily, reaching values as high as $10^{5}$ for $c=31 \mu \mathrm{m}$. However, these huge figuresof-merit are unfounded because the exact same property that gives high dispersions also gives high bend sensitivity and thus implies in the use of large diameter spools, which is completely impractical. The bend loss arises because barriers with large radius force a significant amount of energy to travel close to the edge of the fiber. A throughout study is necessary in order to find the optimum value of $c$ that gives a high dispersion and does not produce a significant bend loss. 


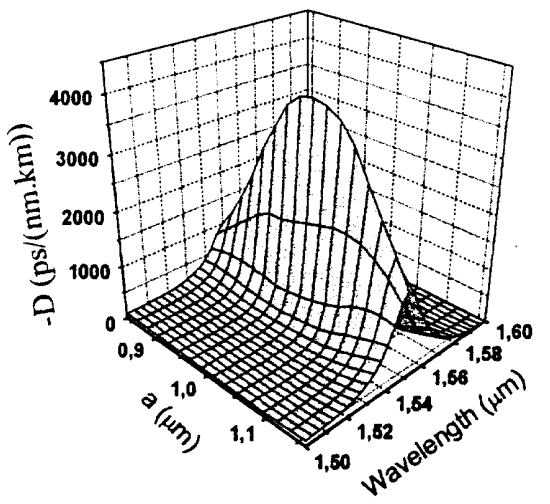

(a)

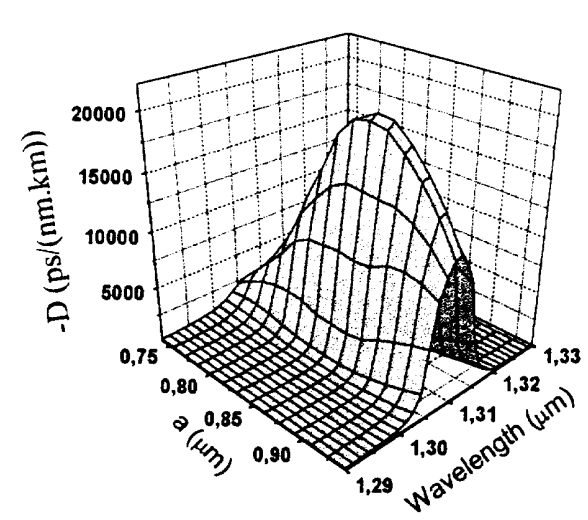

(b)

Figure 4. Wavelength-dependent dispersion curves for different pairs (a,b) at (a) $1.55 \mu \mathrm{m}$ and (b) $1.31 \mu \mathrm{m}$.

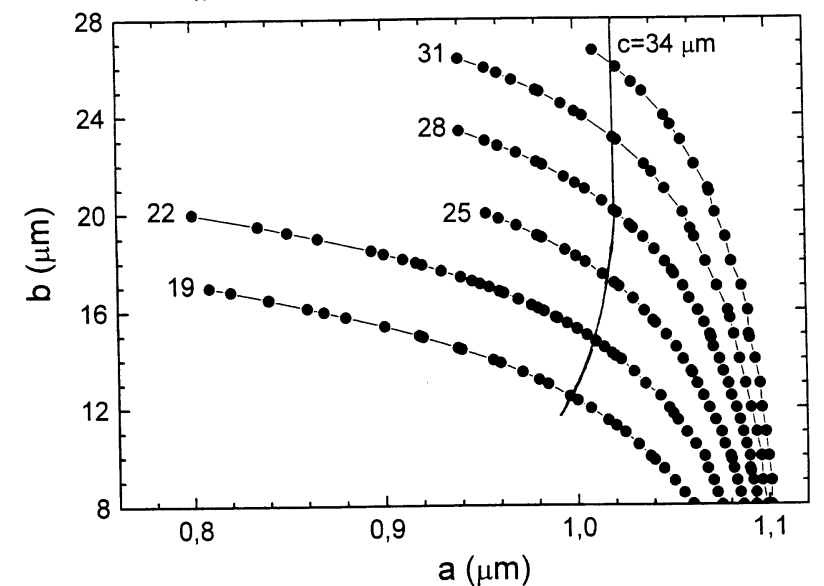

Figure 5. Pairs of radius $a$ and $b$ that provides the maximum dispersion at $1.55 \mu \mathrm{m}$ for different values of $c$. The nearly vertical curve corresponds to pairs that give the highest dispersion.

Another point to be considered is the spatial field distribution of the supermodes $\mathrm{HE}_{11}$ and $\mathrm{HE}_{12}$. They have modal profiles with a central peak, which are quite similar for both modes and two lateral peaks, also similar in profile but with opposite signals. As already discussed by Boucouvalas [8], these two supermodes are able to describe the fundamental modes of rod fibers as well of tube fibers, by either adding or subtracting them. When the modes $\mathrm{HE}_{11}$ and $\mathrm{HE}_{12}$ are added, the lateral peaks cancel and the final result is a Gaussianlike distribution as is the case of the fundamental mode of a rod fiber. By taking their difference, the central peaks will be canceled while the lateral peaks will be added, resulting in the profile of the fundamental mode of a tube [8]. Accordingly, it is possible to devise a way to excite the fundamental mode of the coaxial fiber from the fundamental mode of a standard SMF with a taper made with a coaxial fiber. When the SMF fundamental mode is launched into the taper, will be decoupled in two or more modes, in response to the lack of longitudinal invariance. In the case of an adiabatic taper, only the modes $\mathrm{HE}_{11}$ and $\mathrm{HE}_{12}$ will be excited, but just a small power (less then $10 \%$ ) will be located in the $\mathrm{HE}_{12}$ mode and the remaining will be transported by the $\mathrm{HE}_{11}$ mode [12]. Therefore, there will a dispersion compensation for most of the signal propagating in the coaxial fiber. Since the mode $\mathrm{HE}_{12}$ has a positive dispersion it will be spreaded giving rise to a negligibly small pedestal. When the light reaches the end of the coaxial fiber, another taper of appropriate length, will cause the opposite effect regenerating the fundamental mode of the SMF rod fiber.

\section{Conclusions}

We have analyzed the performance of a dispersion compensating coaxial fiber as a function of two of its geometric parameters, the rod radius and the outer gap radius. We found pairs $(a, b)$ that provide the highest efficiency at a fixed wavelength and studied how the choice of these pairs affect the general shape of the wavelength-dependent dispersion curve. Our analysis was carried out by taking the second derivative of the effective index of refraction obtained from transcendental equations arising when the wave equation is solved under the linearly polarized approximation. The results 
were found to be in agreement with those of Thyagarajan et al. [4]. The modal field distribution for $\mathrm{HE}_{11}$ and $\mathrm{HE}_{12}$ were calculated, although not presented here, and the mechanism of excitation of the coaxial fiber fundamental mode from the fundamental mode of a rod SMF with a taper was discussed.

\section{Acknowledgments}

This work was supported by Fundação de Amparo à Pesquisa do Estado de São Paulo (FAPESP), Conselho Nacional de Desenvolvimento Científico e Tecnológico (CNPq)/RHAE and program PRONEX, under the grant 4.1.96.0935.00.

\section{References}

[1] H. Izadpanah, C. Lin, J. L. Gimlett, A. J. Antos, D. W. Hall and D. K. Smith, Electron. Lett. 28, 1469 (1992).
[2] A. J. Antos, and D. K. Smith, J. Lightwave Technol. 12, 1739 (1994).

[3] U. Peschel, T. Peschel, and F. Lederer, Appl. Phys. Lett. 67, 2111 (1995).

[4] K. Thyagarajan, R. K. Varshney, P. Palai, A. K. Ghatak and I. C. Goyal, IEEE Photon. Technol. Lett. 8, 1510 (1996).

[5] K. C. Kao, and G. A. Hockham, Proc. IEE. 113, 1151 (1966).

[6] M. M. Z. Kharadly, and J. E. Lewis, Proc. Inst. Elec. Eng. 116, 214 (1969).

[7] E. Sharma, A. Sharma, and I. C. Goyal, IEEE J. Quant. Elect. QE-18, 1484 (1982).

[8] A. C. Boucouvalas, J. Lightwave Technol. LT-3, 1151 (1985).

[9] F. D. Nunes, C. A. de S. Melo, and H. Filomeno da Silva Filho, Appl. Optics 35, 388 (1996).

[10] M. J. Adams, An Introduction to Optical Waveguides, John Wiley \& Sons, pp. 213 (1981).

[11] D. Marcuse, Light Transmission Optics, Princeton, NJ: Van Nostrand, pp. 484, (1972).

[12] J. D. Love, IEE. Proceedings 136, 225 (1988). 